\title{
Immunotherapy and Interventional Radiology (IR): Time to Change the Paradigm-Keynote Lecture PAIRS 2021
}

\author{
Thierry de Baère ${ }^{1,2}$ \\ ${ }^{1}$ Interventional Radiology, Gustave Roussy, Villejuif, France \\ 2Université Paris-Saclay, Villejuif, France
}

Arab J Intervent Radiol 2021;5:1-2.

It is time to change the paradigm by considering immunotherapy as essential part of cancer treatment. As you know, PD-L1 and other immune checkpoint inhibitors are of the new and promising pillars of cancer treatments. These treatments are agnostic of the type of cancer disease, even if they are more efficient in melanoma and nonsmall cell cancer than in colorectal cancer, but in some way, they can work in any type of cancer as far as the disease is immune hot. ${ }^{1}$ We have to acknowledge that immunotherapy treatments can dramatically change the outcome for a subpopulation of patients with improved response rate and prolonged overall survival. ${ }^{2}$ Interestingly, the "tail of the curve" is flat and corresponds to a percent of patient which will survive for many years. However, we have to acknowledge that this tail of the curve is at maximum 20 to $30 \%$ of the cancer population, and we have to improve the global outcomes of cancer, namely, in the subpopulation of patients which does not respond to immunotherapies.

To improve efficacy, several combinations have been evaluated, including combination of PD-1, PD-L1 with vascular endothelial growth factor (VEGF) inhibitors, chemotherapy, CTLA-4 checkpoint inhibitors, so a lot of these combinations are under investigation. For example, atezolizumab + bevacizumab was demonstrated to be superior to sorafenib for advanced hepatocellular carcinoma in the Imbrave150 study and is today the standard of care. ${ }^{3}$ Combination of PD-L1 inhibitors with chemotherapy in head and neck squamous cell carcinoma (HNSCC) have demonstrated $44 \%$ response rate that compares favorably with pembrolizumab alone with $20 \%$, while chemotherapy demonstrated $40 \%$ response rate, but it is noteworthy that the duration of response for pembrolizumab alone is longer than chemo alone or combination, with
Address for correspondence Thierry de Baere, MD, PhD, Department of Interventional Radiology, Gustave Roussy Institute, 114 rue Edouard Vaillant, Villejuif Cedex, 94805, France (e-mail: thierry.debaere@gustaveroussy.fr).

chemotherapy possibly altering immune cells which does not aid in prolonged response. ${ }^{4}$ Combination of two different checkpoints, inhibitor PD-1 plus CTL-A4, comes at the cost of increased toxicity from 27 to $60 \% 5$

The concept of human intratumoral immunotherapy involves producing a local priming of tumor, with intratumoral injection of different compounds along with the expectation of on-tumor effects, which will be distant from the site of injection. Such in situ immunization has major advantages over cancer vaccine, that is, a one off-the-shelf product that might fit all patients, which avoids tumor sampling and patient per patient tumor vaccine harvesting.

A comparison of intratumoral versus intravenous administration of ipilimumab in combination with systemic administration of nivolumab in patients with metastatic melanomas is under evaluation (NCT028575669). Intratumoral administration of toll-like receptors (TLR)3, 4, 7/ 8 and 9 agonists is under evaluation for the treatment of solid and hematologic malignancies. Immunomodulation with an oncolytic peptide LTX-315, which induces a malignant cell death and elicits anticancer immune responses, has been demonstrated to rapidly reprogram the tumor microenvironment (NCT01986426). ${ }^{6}$ Recently, an oncolytic virus, talimogene laherparepvec (T-VEC), has been approved by the Food and Drugs Administration (FDA) for the treatment of unresectable cutaneous, subcutaneous, and nodal lesions in patients with recurrent melanoma after initial surgery. Recently, T-VEC has demonstrated a tolerable safety profile with ipilimumab, and the combination appeared to have greater efficacy than either T-VEC or ipilimumab monotherapy. ${ }^{7}$ Its added value when used in combination with anti-PD-1 is currently being tested in a randomized phase 3 study (NCT02263508). published online

June 24, 2021
DOI https://doi.org/

$10.1055 / \mathrm{s}-0041-1731604$ ISSN 2542-7075 (c) 2021. The Pan Arab Interventional Radiology Society

This is an open access article published by Thieme under the terms of the Creative Commons Attribution-NonDerivative-NonCommercial-License, permitting copying and reproduction so long as the original work is given appropriate credit. Contents may not be used for commercial purposes, or adapted, remixed, transformed or built upon. (https://creativecommons.org/licenses/by-nc-nd/4.0/).

Thieme Medical and Scientific Publishers Private Ltd. A-12, Second Floor, Sector -2, NOIDA -201301, India 
Interventional radiologists have to be part of this research, because they are the best qualified for providing safe, accurate tumoral injections, ideally under image guidance. They can help to answer many unsolved questions such as: Does some tumor location need to be prioritized for injection because of better outcomes? What is the ideal imaging for targeting active tumor cells and the microenvironment while avoiding necrosis? Today, most of the target lesions will have to be injected several times over a few months and, as interventional radiologists (IRs), we must select the adequate target and search for possible delivery platforms. Technical aspects such as needle size, needle type (end hole, side all, etc.) are controlled by IRs and also need research. ${ }^{8}$

Close collaboration between medical oncologists, diagnostic radiologists, IRs, and planning nurses are key to successful implementation of intratumoral immunotherapy trials and clinical practice, to the point that a multidisciplinary tumor board dedicated to this practice is recommended. ${ }^{9}$

Many questions remain to be answered, and IRs must be part of this research: dose by body weight versus dose per tumor? small volume versus large volumes? Where is the drug effectively delivered? Does it stay within the tumor? for how long? Do we need X-ray visible drug? Do we need delivery

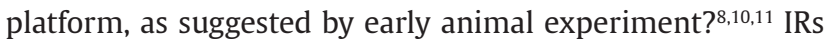
must investigate other intratumoral routes such as intralymphatic and intra-arterial. Maybe in the future, we can inject in size of the particle theory. There are many options that we have to embrace, and we have to be part of this broad delivery story for immune-oncology.

Finally, as diagnostic radiologists, we need to thoroughly evaluate intratumoral immunotherapy, as separately evaluating the injected lesions and not-injected lesions distant from the location of injection is a major point. We need to better understand our new treatment, and realize that response evaluation criteria in solid tumors (RECIST) system is not enough when dealing with local therapy, with the potential of a global effect. Intratumoral RECIST, called itRECIST, criteria have been recently reported and need to be applied. ${ }^{12}$

Both interventional oncology and immuno-oncology use the same abbreviation IO. It is important that both IOs can collaborate in research to provide the best to our patients.

\section{Conflict of Interest}

Dr. de Baere reports personal fees from Guerbet, and grants and personal fees from Terumo, outside the submitted work.

\section{References}

1 Hirsch L, Zitvogel L, Eggermont A, Marabelle A. PD-Loma: a cancer entity with a shared sensitivity to the PD-1/PD-L1 pathway blockade. Br J Cancer 2019;120(1):3-5

2 Antonia SJ, Borghaei H, Ramalingam SS, et al. Four-year survival with nivolumab in patients with previously treated advanced non-small-cell lung cancer: a pooled analysis. Lancet Oncol 2019;20(10):1395-1408

3 Finn RS, Qin S, Ikeda M, et al. IMbrave150 Investigators. Atezolizumab plus bevacizumab in unresectable hepatocellular carcinoma. N Engl J Med 2020;382(20):1894-1905

4 Burtness B, Harrington KJ, Greil R, et al. KEYNOTE-048 Investigators. Pembrolizumab alone or with chemotherapy versus cetuximab with chemotherapy for recurrent or metastatic squamous cell carcinoma of the head and neck (KEYNOTE-048): a randomised, open-label, phase 3 study. Lancet 2019;394(10212):1915-1928

5 Larkin J, Chiarion-Sileni V, Gonzalez R, et al. Five-year survival with combined nivolumab and ipilimumab in advanced melanoma. N Engl J Med 2019;381(16):1535-1546

6 Yamazaki T, Pitt JM, Vétizou M, et al. The oncolytic peptide LTX-315 overcomes resistance of cancers to immunotherapy with CTLA4 checkpoint blockade. Cell Death Differ 2016;23(6):1004-1015

7 Puzanov I, Milhem MM, Minor D, et al. Talimogene laherparepvec in combination with ipilimumab in previously untreated, unresectable stage IIIB-IV melanoma. J Clin Oncol 2016;34(22):2619-2626

8 Muñoz NM, Williams M, Dixon K, et al. Influence of injection technique, drug formulation and tumor microenvironment on intratumoral immunotherapy delivery and efficacy. J Immunother Cancer 2021;9(2):e001800

9 Champiat S, Tselikas L, Farhane S, et al. Intratumoral immunotherapy: from trial design to clinical practice. Clin Cancer Res 2021;27(3):665-679

10 Tselikas L, Champiat S, Sheth RA, et al. Interventional Radiology for Local Immunotherapy in Oncology. Clin Cancer Res 2021;27(10):2698-2705

11 Tselikas L, de Baere T, Isoardo T, et al. Pickering emulsions with ethiodized oil and nanoparticles for slow release of intratumoral anti-CTLA4 immune checkpoint antibodies. J Immunother Cancer 2020;8(1):e000579

12 Goldmacher GV, Khilnani AD, Andtbacka RHI, et al. Response criteria for intratumoral immunotherapy in solid tumors: itRECIST. J Clin Oncol 2020;38(23):2667-2676 